\title{
EVALUACIÓN Y DIAGNÓSTICO IMAGENOLÓGICO DE LAS REABSORCIONES RADICULARES INTERNAS: UNA REVISIÓN DE LITERATURA
}

\author{
INTERNAL RADICULAR RESORPTION, EVALUATION AND IMAGENOLOGICAL \\ DIAGNOSIS: A REVIEW OF THE LITERATURE
}

Bianca Guerra Santa Cruz'

biancaguerra@hotmail.com

ORCID: 0000-0002-9613-4884

\section{RESUMEN}

La reabsorción radicular interna es la pérdida de dentina intrarradicular por acción odontoclástica. El proceso es causado principalmente por una infección crónica, trauma, avulsión o reimplantación. La reabsorción radicular interna se clasifica en inflamatoria y de reemplazo, ambas implican pérdida de dentina radicular; la reabsorción radicular de reemplazo es la respuesta a una reparación frustrada de la reabsorción radicular inflamatoria, con la posterior deposición de un tejido duro metaplásico. Se ha demostrado la superioridad de la tomografía computarizada de haz cónico en el diagnóstico de las cavidades pequeñas de RRI, aunque no todas las imágenes de la tomografía computarizada de haz cónico ofrecen la misma resolución. Además del protocolo FOV en la tomografía computarizada de haz cónico, el tamaño de vóxel puede influenciar también en el diagnóstico de las reabsorciones radiculares internas. Imágenes 2D de cavidades grandes de reabsorción radicular interna (mayores a 0,6 $\mathrm{mm}$ de diámetro) han demostrado igual sensibilidad y especificidad en comparación con las imágenes 3D. Actualmente, no existen protocolos para la adquisición imagenológica de las diferentes condiciones de las reabsorciones radiculares internas.

Palabras clave: reabsorción radicular interna, 2D, 3D, tomografía computarizada de haz cónico, vóxel

\section{ABSTRACT}

Internal root resorption is the loss of intraradicular dentine by odontoclastic action. This process is principally caused by chronic infection, trauma, avulsion and reimplantation. Internal root resorption is classified as inflammatory and replacement, with both implying loss of intraradicular dentine. Replacement root resorption is the reaction of an unsuccessful repair of inflammatory radicular resorption with the subsequent deposition of metaplastic hard tissue. Although cone beam computed tomography (CBCT) been shown to be effective in the diagnosis of small internal root resorption

Citar como: Guerra B. Evaluación radiológica de la reabsorción radicular interna. Una revisión de la literatura. Rev Cient Odontol (Lima). 2019; 7 (1): 103-112. 
cavities, not all CBCT images provide the same resolution. In addition to the field of view protocol, voxel size can also influence the diagnosis of internal root resorption. 2D images of large cavities of internal root resorption (larger than $0.6 \mathrm{~mm}$ of diameter) have shown to have the same sensivity and specificity as 3D images. Currently there are no imaging acquisition protocols for studying different aspects of internal root resorption.

Keywords: internal root resorption, 2D, $3 \mathrm{D}$, cone beam computed tomography, voxel

\section{INTRODUCCIÓN}

La reabsorción radicular interna es un proceso de pérdida de la dentina circundante al conducto radicular debido a la actividad de odontoclastos gigantes multinucleados adyacentes al tejido granulado en la pulpa, a partir de la inflamación crónica pulpar ( $\left.{ }^{1}\right)$. Las RRI generalmente son asintomáticas $\left({ }^{2}\right)$; clínicamente, a veces se manifiestan a nivel de la corona dentaria como una mancha rosada debido al tejido hiperplásico de la pulpa en el área de reabsorción ( $\left.{ }^{3-5}\right)$. La etiología de las RRI es multifactorial6, aunque principalmente son relacionadas a lesiones dentales traumáticas $\left({ }^{7}\right)$, inflamación bacteriana continua $\left({ }^{8}\right)$, la avulsión y la reimplantación $\left({ }^{9,10}\right)$. Se ha reportado inclusive casos de $\mathrm{RRI}$ relacionados con el virus del herpes zóster $\left({ }^{11}\right)$.

La RRI y la reabsorción radicular externa no son procesos patológicos iguales, tienen diferente etiología; por ello, es importante diferenciarlas para determinar el protocolo de tratamiento adecuado. Resulta útil en el diagnóstico diferencial de la RRI y la RRE, la técnica radiográfica de localización de Clark ( ${ }^{12}$ ). La RRI inflamatoria es el tipo más frecuente $y$, radiográficamente, se observa el conducto radicular de forma ovalada o circular de bordes lisos y definidos $\left({ }^{5}\right)$. Esta apreciación bidimensional resulta limitada para diagnosticar el daño real que la $R R I$ causa en la raíz dental $\left({ }^{13}\right)$. La gravedad, localización y tipo de $R R I$ resulta inexacta radiográficamente $\left({ }^{14-16}\right)$. La cantidad de pared dentinaria restante en sentido buco lingual en la lesión de la RRI, con o sin perforación externa, solo es posible observarla con CBCT $\left({ }^{13,17,18}\right)$. Cavidades pequeñas, de $0,6 \mathrm{~mm}$ de diámetro y menos de $0,3 \mathrm{~mm}$ de profundidad, no son detectables en las radiografías periapicales convencionales $\left({ }^{19}\right)$.

Se ha demostrado la superioridad de la $\mathrm{TCHC}$ en el diagnóstico de las RRI, aunque no todas las imágenes con TCHC ofrecen la misma resolución $\left.{ }^{20}\right)$. Algunos estudios mostraron una tendencia hacia resultados más precisos de las $R R I$ con mejores resoluciones usando $\mathrm{TCHC}$ con vóxeles específicos $\left({ }^{13,20,21}\right)$. El tamaño del vóxel en la TCHC es equivalente a la resolución de píxeles en imágenes radiográficas 2D; un tamaño de vóxel de 0,085 $\mathrm{mm}$ son equivalentes a 300 píxeles por pulgada $\left({ }^{22,23}\right)$. Imágenes tridimensionales exactas y precisas del área y la extensión de la lesión de la RRI pueden modificar la secuencia habitual en el plan de tratamiento quirúrgico o no quirúrgico para esta patología $\left({ }^{18}\right)$.

Según la Sociedad Europea de Endodoncia, actualmente se tiene como norma el uso de la TCHC en el manejo de las $R R$, y la recomendación número 12 indica el uso de la TCHC en el diagnóstico diferencial entre la $R R I$ y la RRE, así como en la determinación del apropiado tratamiento y pronóstico de las $R R$ ${ }^{(24)}$. Sin embargo, actualmente, no existe estandarización en el protocolo de adquisición de imágenes $3 \mathrm{D}$ de las RRI. Por consiguiente, el objetivo principal de este trabajo es revisar información que nos ofrece la literatura contemporánea con 
respecto al diagnóstico imagenológico de las RRI que favorecen una mejor interpretación de esta alteración.

\section{DESCRIPCIÓN E HISTOLOGIA DE LAS REABSORCIONES RADICULARES INTERNAS}

La RRI es la pérdida progresiva de la dentina con sustitución del tejido de la pulpa por odontoclastos que son células gigantes granulomatosas que reabsorben dentina ${ }^{25}$ ). Los odontoclastos y osteoclastos son morfológicamente similares, ambos de una manera similar reabsorben sus tejidos diana $\left({ }^{4}\right)$. Según estudios $\left({ }^{26,27}\right)$, células dendríticas inmaduras son precursoras de los osteoclastos; por consiguiente, células dendríticas de la pulpa dental probablemente sean precursoras de odontoclastos. El comienzo del proceso de reabsorción se da por los cambios vasculares en la pulpa, la tensión de oxígeno se incrementa y el pH disminuye, por consiguiente numerosos macrófagos son atraídos al sitio $\left({ }^{3}\right)$

La RRI se asocia principalmente con el trauma dental $\left.{ }^{(28,29}\right)$, en esta situación el proceso de reabsorción se presenta dentro de unas pocas semanas o meses después del evento. En esta situación de trauma después de la hemorragia intrapulpar se presentan coágulos cuyo tejido conectivo puede presentar metaplasia para formar tejido de granulación $\left.{ }^{(30}\right)$, activándose posteriormente los odontoclastos, que son las células responsables de la RRI $\left({ }^{5}\right)$.

Los bordes en cepillo y las zonas claras son las dos especializaciones de la membrana celular de los odontoclastos $\left({ }^{31}\right)$. Las zonas claras tienen abundantes microfilamentos que se unen con mucha afinidad a la dentina, unión que permite el sellado de los bordes en cepillo. A través de esta, se bombean protones y se permite el paso de las enzimas secretadas por las organelas, lo que crea un ambiente ácido que disuelve minerales (entre otras, calcio). Estos minerales son endocitados y llevados a la porción central de los bordes en cepillo y, por medio de vesículas, son llevados al torrente sanguíneo ( ${ }^{32}$ ). Progresivamente, el tejido pulpar se va necrosando, lo que limita el proceso de la RRI al actuar como sistema de protección del conducto radicular.

\section{ETIOLOGÍA, \\ CARACTERISTICAS \\ CLINICAS Y \\ CLASIFICACIÓN DE LAS RRI}

La RRI suele ser asintomática y generalmente es causada por una infección crónica o un trauma $\left({ }^{7,8}\right)$. También se ha considerado a la avulsión y la reimplantación como principal factor desencadenante de las RR $\left({ }^{9,10}\right)$. Otras causas relacionadas son la eliminación parcial de la pulpa, la ortodoncia, la caries, el recubrimiento pulpar con hidróxido de calcio o pulpotomía, el calor extremo y un diente agrietado, quistes dentígeros, neoplasias y factores idiopáticos $\left({ }^{33,34}\right)$. Se ha relacionado inclusive con el virus del herpes zóster $\left({ }^{11}\right)$.

Clínicamente, la afección es, por lo general, asintomática; sin embargo, puede incluir la presencia de una zona rojiza, una mancha rosada que representa el tejido de granulación que se ve a través del área reabsorbida, a nivel de la corona clínica $\left({ }^{6}\right)$ (figura 1). La RRI se puede clasificar como inflamatoria y de reemplazo $\left.{ }^{35}\right)$, tipos de reabsorción que implican una pérdida continua de dentina radicular. La RRI inflamatoria puede ser perforante o no perforante, y la RRI de reemplazo implica, además de la zona de reabsorción en esta área, la posterior deposición de tejido duro que se parece al hueso o al cemento, pero no a la dentina $(4,36,37$ ) (figura 2). 


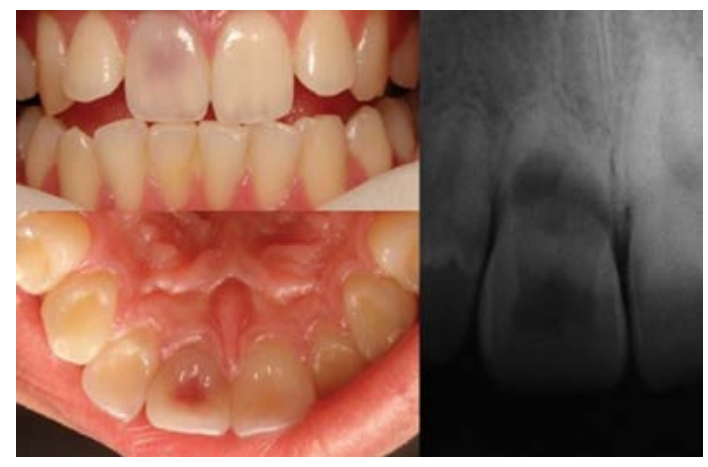

FIGURA 1. LA REABSORCIÓN RADICULAR INTERNA, CLÍNICAMENTE, SE OBSERVA A VECES A NIVEL DE LA CORONA DENTARIA COMO UNA MANCHA ROSADA.

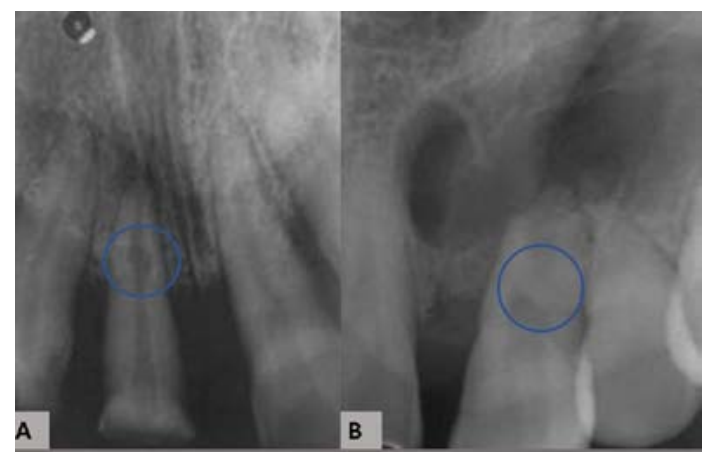

Figura 2. A. REABSORCIÓn RADICULAR INTERNA INFLAMATORIA. B. REABSORCIÓN RADICULAR INTERNA DE REEMPLAZO, QUE IMPLICA LA POSTERIOR DEPOSICIÓN DE TEJIDO DURO PARECIDO A HUESO O CEMENTO EN LA ZONA DE REABSORCIÓN.

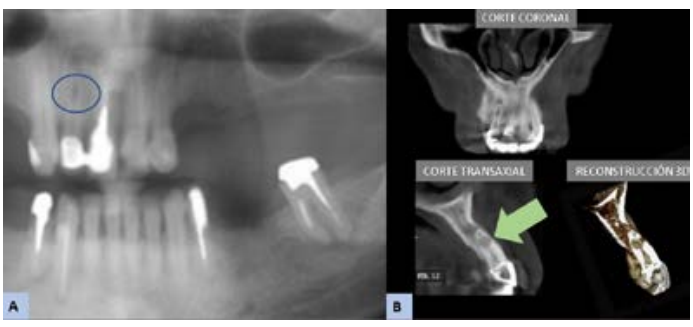

FIgURA 3. A. IMAGEN 2D: PIEZA 1.2 cON RRI INFLAMATORIA. B. IMAGEN 3D: EN EL CORTE TRANSAXIAL SE OBSERVA EL DAÑO REAL DE LA RRI EN SENTIDO BUCOLINGUAL.

\section{DIAGNOSTICO IMAGENOLÓGICO 2D Y 3D DE LAS RRI}

Las $R R I$ se relacionan principalmente con situaciones de trauma dental, tras este episodio a las semanas debería considerarse como primer examen de diagnóstico a la TCHC, pues se ha evidenciado su alta precisión en el diagnóstico de pequeñas cavidades de reabsorción en comparación con la radiografía convencional $(2,21)$.

Actualmente, dentro del manejo de las RRI, ya se tiene como consenso el uso de la TCHC, según la Sociedad Europea de Endodoncia, que indica como norma el uso de la TCHC para la gestión de las reabsorciones radiculares $\left({ }^{24}\right)$. Dicha declaración se basa en la corriente de evidencia científica sobre cuándo utilizar TCHC en endodoncia. Sin embargo, actualmente, dicho consenso no específica el protocolo de adquisición de imágenes con TCHC para el diagnóstico de las RRI. El tratamiento y el pronóstico de la RRI se basan en la determinación precisa y exacta de la extensión real de la cavidad de la RRI (figura 3). Un diagnóstico precoz de esta patología evita la pérdida de la estructura dental y conduce a un buen pronóstico del tratamiento $\left({ }^{38}\right)$.

Las cavidades pequeñas de las RRI, de $0,6 \mathrm{~mm}$ de diámetro y menos de $0,3 \mathrm{~mm}$ en profundidad, no son detectables en las radiografías periapicales convencionales $\left({ }^{19,39,40}\right)$. Sin embargo, lesiones grandes de RRI, radiográfica y tomográficamente, tienen precisión diagnostica similar $\left({ }^{41-44}\right)$.

Las $R R I$ inflamatoria en las radiografías convencionales se observan con los márgenes lisos y claramente definidos. Las paredes del sistema de conductos radiculares pueden parecer como un globo hinchado $\left({ }^{45,46}\right)$. La lesión de las RRE tiene los márgenes irregulares y mal definidos, es asimétrica, su radiodensidad no es uniforme, y si la lesión se superpone sobre el sistema de conductos debería ser posible seguir las paredes del conducto inalterados a través del área de defecto $\left({ }^{47}\right)$ (figura 4). En las RRI, se observa que la cámara pulpar y el canal no se pueden seguir a través de la lesión, la distribución del conducto radicular es simétrica; pero puede ser excéntrico y la radiotransparencia es de 


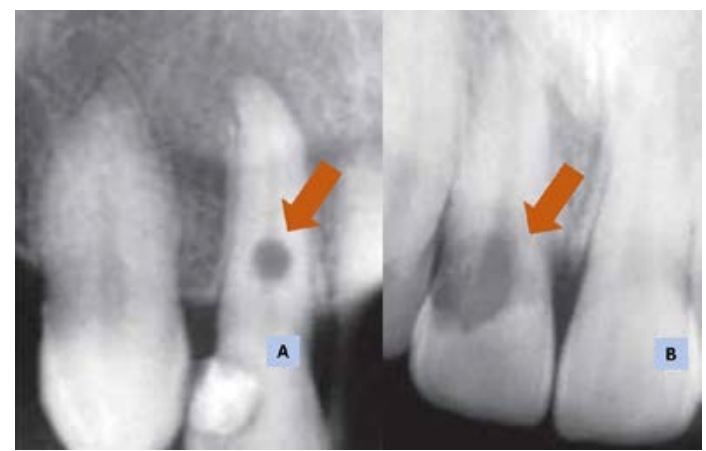

FIGURA 4. A. REABSORCIÓN RADICULAR INTERNA INFLAMATORIA: EL CANAL RADICULAR NO SE PUEDE SEGUIR A TRAVÉS DE LA LESIÓN. B. REABSORCIÓN RADICULAR INTERNA DE REEMPLAZO:

EL CANAL RADICULAR SE PUEDE SEGUIR A TRAVÉS DE LA LESIÓN.

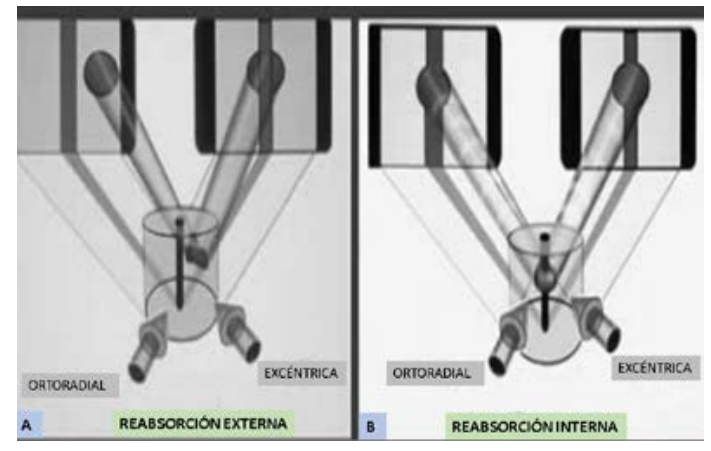

FIGURA 5. DIAGNÓSTICO DIFERENCIAL RADIOGRÁFICAMENTE ENTRE LA RRE Y LA RRI. A. RRE: LA LESIÓN SE DESPLAZA DE LUGAR TRAS UNA INCIDENCIA RADIOGRÁFICA EXCÉNTRICA. B. RRI: LA LESIÓN SE MANTIENE EN SU LUGAR TRAS UNA INCIDENCIA RADIOGRÁFICA EXCÉNTRICA.

densidad uniforme. Al realizar el diagnóstico diferencial radiográfico entre la RRI y la RRE con la técnica de localización de Clarck tras una incidencia excéntrica la lesión de la RRI se mantiene en el mismo lugar $\left({ }^{12}\right)$; mientras que en las RRE se desplaza (figura 5 ).

Varios estudios $(12,17,18,21,28,41,48,49)$, demuestran la superioridad en el diagnóstico de las RRI usando TCHC, específicamente en el diagnóstico de cavidades pequeñas de RRI $\left({ }^{17}\right)$, mientras que la radiografía convencional, digital y $\mathrm{TCHC}$ resultaron igualmente eficaces en el diagnóstico de cavidades grandes de RRI $\left({ }^{41}\right)$.
Se evaluó la TCHC y su diagnóstico en lesiones de RRI con perforación y sin perforación. Como resultado, la TCHC tuvo mejor precisión de diagnóstico en ambas situaciones $\left({ }^{13}\right)$. La TCHC obtuvo mejores resultados que la radiografía digital en el diagnóstico de la RRI inflamatoria después de un trauma dental en dientes ya endodonciados $\left({ }^{12}\right)$. Se ha demostrado que la RRI por sustitución fue diagnosticada con igual precisión mediante las radiografías periapicales y la TCHC $\left({ }^{12}\right)$.

En el seguimiento de la lesión de las RRI, resulta importante la estandarización en las directrices para adquirir las imágenes con la $\mathrm{TCHC}$, para no realizar interpretaciones falsas de su extensión, ya que esta situación puede crear decisiones erróneas en su plan de tratamiento $\left.{ }^{(20}\right)$. Algunos estudios mostraron una tendencia hacia resultados más precisos en el diagnóstico de las RRI con mejores resoluciones usando TCHC con vóxeles específicos $\left({ }^{13,20,21}\right)$.

La TCHC ofrece imágenes de múltiples planos en resolución submilimétrica $\left({ }^{50}\right)$. Los diferentes tomógrafos actuales en el mercado difieren en características técnicas como la resolución espacial representada por el tamaño de vóxel $\left({ }^{51}\right)$ (figura 6). Cuanto menor sea el campo de visión y el tamaño de vóxel, mejor es la resolución espacial $\left({ }^{22,52}\right)$. La literatura confirma la influencia del tamaño de vóxel en la resolución de imágenes para el diagnóstico de diferentes patologías $\left.{ }^{52-56}\right)$. La resolución de imágenes $2 \mathrm{D}$ representada por los pixeles es equivalente al tamaño de vóxel en imágenes 3D (300 pixeles por pulgada corresponden a $0,085 \mathrm{~mm}$ del tamaño de un vóxel) $\left({ }^{23}\right)$.

La evaluación tridimensional de las RRI puede modificar el plan de tratamiento habitual para este tipo de lesión $\left({ }^{18,28}\right)$. Las técnicas de termoplastificación de gutapercha $u$ ondas continuas son el 

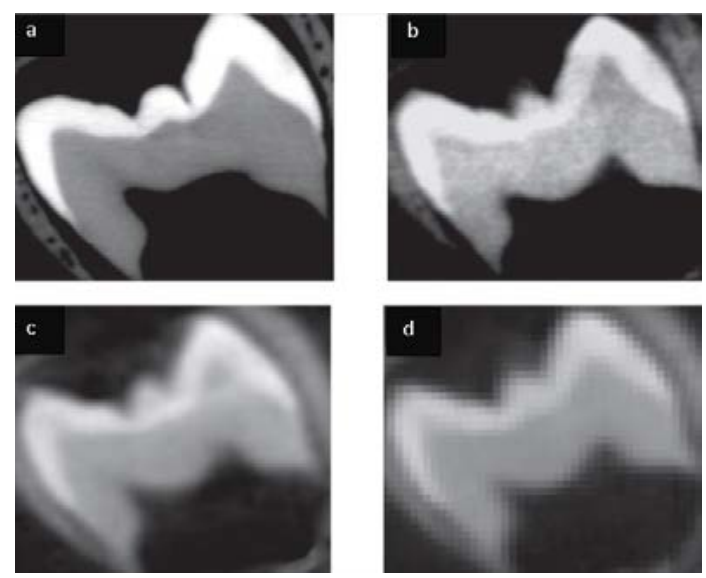

FIGURA 6.

A. IMAgen 3D CON tamaño DE VóXel DE $41 \mathrm{Mm}$. B. IMAGEN 3D CON TAMAÑO DE VÓXEL DE $76 \mathrm{MM}$. C. IMAGEN 3D CON TAMAÑo DE VÓXEL DE 200 MM. D. IMAGEN 3D CON TAMAÑO DE VÓXEL DE 300 MM.

procedimiento de elección frecuente en la obturación de conductos con cavidades de RRI; sin embargo, si las paredes de estas cavidades son muy delgadas y superan el umbral de la temperatura permitida en la superficie externa de la raíz ocasionarían destrucción del tejido periodontal. Ahí la importancia de la TCHC en el diagnóstico de la extensión real de las $R R I$ para la determinación de la técnica de obturación a elegir $\left({ }^{57}\right)$.

\section{CONCLUSIONES}

Esta revisión de literatura está orientada principalmente a contribuir con el radiólogo en la formulación de las directrices para la adquisición imagenológica de las RRI. Imágenes 2D de cavidades grandes de RRI (mayores a 0,6 $\mathrm{mm}$ de diámetro) han demostrado igual sensibilidad y especificidad en comparación con las imágenes 3D. La TCHC es superior a la radiografía en el diagnóstico de las RRI, especialmente en cavidades pequeñas (menores a 0,6 $\mathrm{mm}$ de diámetro) y ofrecen, además, más información sobre su extensión real. Además del protocolo FOV en la TCHC, el tamaño del vóxel puede influenciar también en el diagnóstico de las RRI, la literatura aún escasa en este tópico indica en promedio el uso de $0,100 \mathrm{~mm}$ y máximo $0,200 \mathrm{~mm}$ de vóxel para un diagnóstico con mayor resolución de las RRI. Actualmente, no existen protocolos para la adquisición imagenológica de las diferentes condiciones de las RRI.

Contribución del autor: Bianca Guerra Santa Cruz participó en la concepción del artículo, la revisión de datos, su redacción y aprobación de la versión final.

Fuente de financiamiento: Autofinanciado

Conflicto de Interés: La autora declara no tener conflictos de interés de ningún tipo. 


\section{REFERENCIAS BIBLIOGRÁFICAS}

1. Nilsson E, Bonte E, Bayet F, Lasfargues JJ. Management of internal root resorption on permanent teeth. Int J Dent. 2013;2013:929486.

2. Kamburoglu K, Kursun S, Yuksel S, Oztas B. Observer ability to detect ex vivo simulated internal or external cervical root resorption. J Endod. 2011; 37 (2): 168-75.

3. Thomas P, Krishna Pillai R, Pushparajan Ramakrishnan B, Palani J. An insight into internal resorption. ISRN Dent. 2014; 2014: 759326.

4. Patel S, Ricucci D, Durak C, Tay F. Internal root resorption: a review. J Endod. 2010; 36 (7): 1107-21.

5. Petel R, Fuks A. Pink Spot - Literature Review and Case Report. J Clin Pediatr Dent. 2016; 40 (5): 353-5.

6. Mittal S, Kumar T, Mittal S, Sharma J. "Internal root resorption: An endodontic challenge": A case series. J Conserv Dent. 2014; 17 (6): 590-3.

7. Caliskan MK, Turkun M. Prognosis of permanent teeth with internal resorption: a clinical review. Endod Dent Traumatol. 1997; 13 (2): 75-81.

8. Gabor C, Tam E, Shen Y, Haapasalo M. Prevalence of internal inflammatory root resorption. J Endod. 2012; 38 (1): 24-7.

9. Hecova $\mathrm{H}$, Tzigkounakis $\mathrm{V}$, Merglova $\mathrm{V}$, Netolicky J. A retrospective study of 889 injured permanent teeth. Dent Traumatol. 2010; 26 (6): 466-75.

10. Rocha Lima TF, Nagata JY, de Souza-Filho FJ, de Jesus Soares A. Post-traumatic complications of severe luxations and replanted teeth. J Contemp Dent Pract. 2015; 16 (1): 13-9.

11. Jakovljevic A, Kuzmanovic Pficer J, Dragan IF, Knezevic A, Miletic M, BeljicIvanovic $\mathrm{K}$, et al. The role of varicella zoster virus in the development of periapical pathoses and root resorption: A systematic review. J Endod. 2017; 43 (8): 1230-6.

12. Lima TF, Gamba TO, Zaia AA, Soares AJ. Evaluation of cone beam computed tomography and periapical radiography in the diagnosis of root resorption. Aust Dent J. 2016; 61 (4): 425-31.

13. Khojastepour L, Moazami F, Babaei M, Forghani M. Assessment of Root Perforation within Simulated Internal Resorption Cavities Using Cone-beam Computed Tomography. J Endod. 2015; 41 (9): 1520-3.

14. Liang YH, Li G, Wesselink PR, Wu MK. Endodontic outcome predictors identified with periapical radiographs and cone-beam computed tomography scans. J Endod. 2011; 37 (3): 326-31.

15. D'Addazio PS, Campos CN, Ozcan M, Teixeira HG, Passoni RM, Carvalho AC. A comparative study between cone-beam computed tomography and periapical radiographs in the diagnosis of simulated endodontic complications. Int Endod J. 2011; 44 (3): 218-24. 
16. Patel S, Dawood A, Ford TP, Whaites E. The potential applications of cone beam computed tomography in the management of endodontic problems. Int Endod J. 2007; 40 (10): 818-30.

17. Estrela C, Bueno MR, De Alencar AH, Mattar R, Valladares Neto J, Azevedo $\mathrm{BC}$, et al. Method to evaluate inflammatory root resorption by using cone beam computed tomography. J Endod. 2009; 35 (11): 1491-7.

18. Bhuva B, Barnes JJ, Patel S. The use of limited cone beam computed tomography in the diagnosis and management of a case of perforating internal root resorption. Int Endod J. 2011; 44 (8): 777-86.

19. Andreasen FM, Sewerin I, Mandel U, Andreasen JO. Radiographic assessment of simulated root resorption cavities. Endod Dent Traumatol. 1987; 3 (1): 21-7.

20. Da Silveira PF, Fontana MP, Oliveira HW, Vizzotto MB, Montagner F, Silveira HL, et al. CBCT-based volume of simulated root resorption - influence of FOV and voxel size. Int Endod J. 2015; 48 (10): 959-65.

21. Kamburoglu K, Kursun S. A comparison of the diagnostic accuracy of CBCT images of different voxel resolutions used to detect simulated small internal resorption cavities. Int Endod J. 2010; 43 (9): 798-807.

22. Maret D, Telmon N, Peters OA, Lepage B, Treil J, Inglese JM, et al. Effect of voxel size on the accuracy of $3 \mathrm{D}$ reconstructions with cone beam $\mathrm{CT}$. Dentomaxillofac Radiol. 2012; 41 (8): 649-55.

23. Spin-Neto R, Gotfredsen E, Wenzel A. Impact of voxel size variation on CBCTbased diagnostic outcome in dentistry: a systematic review. J Digit Imaging. 2013; 26 (4): 813-20.

24. Patel S, Durack C, Abella F, Roig M, Shemesh H, et al. European Society of Endodontology position statement: the use of CBCT in endodontics. Int Endod J. 2014; 47 (6): 502-4.

25. Darcey J, Qualtrough A. Resorption: part 1. Pathology, classification and aetiology. Br Dent J. 2013; 214 (9): 439-51.

26. Laperine O, Blin-Wakkach C, Guicheux J, Beck-Cormier S, Lesclous P. Dendriticcell-derived osteoclasts: a new game changer in bone-resorption-associated diseases. Drug Discov Today. 2016; 21 (9): 1345-54.

27. Song L, Dong G, Guo L, Graves DT. The function of dendritic cells in modulating the host response. Mol Oral Microbiol. 2018; 33 (1): 13-21.

28. Perlea $P$, Nistor CC, Iliescu MG, lliescu AA. The use of cone beam computed tomography in the diagnosis and management of internal root resorption associated with chronic apical periodontitis: a case report. Rom J Morphol Embryol. 2015; 56 (1): 223-7.

29. Keinan D, Heling I, Stabholtz A, Moshonov J. Rapidly progressive internal root resorption: a case report. Dent Traumatol. 2008; 24 (5): 546-9.

30. Sikri VK. Root Resorption an Endodontic Enigma. Indian Journal of Comprehensive Dental Care. 2011; 1: 15. 
31. Nanci A, Whitson W, Bone B. Histología oral de Ten Cate: desarrollo, estructura y función. $6^{\mathrm{a}}$ ed. Interamericana; 2003. 122-30 p.

32. Moreno S, Contreras A. Molecular mechanisms involved in bone destruction in periodontitis. Literature review. Revista Clínica de Periodoncia, Implantología y Rehabilitación Oral. 2013; 6 (3): 142-7.

33. Yadav P, Rao Y, Jain A, Relhan N, Gupta S. Treatment of internal resorption with mineral trioxide aggregates: a case report. J Clin Diagn Res. 2013; 7 (10): 2400-1.

34. Vasconcelos KF, Rovaris K, Nascimento EHL, Oliveira ML, Tavora DM, Boscolo FN. Diagnostic accuracy of phosphor plate systems and conventional radiography in the detection of simulated internal root resorption. Acta Odontol Scand. 2017; 75 (8): 573-6.

35. Fernandes $\mathrm{M}$, de Ataide I, Wagle R. Tooth resorption part I - pathogenesis and case series of internal resorption. J Conserv Dent. 2013; 16 (1): 4-8.

36. Consolaro A. The four mechanisms of dental resorption initiation. Dental Press $\mathrm{J}$ Orthod. 2013; 18 (3): 7-9.

37. Wedenberg $\mathrm{C}$, Lindskog $\mathrm{S}$. Evidence for a resorption inhibitor in dentin. Scand $\mathrm{J}$ Dent Res. 1987; 95 (3): 205-11.

38. Esnaashari E, Pezeshkfar A, Fazlyab M. Nonsurgical management of an extensive perforative internal root resorption with calcium-enriched mixture cement. Iran Endod J. 2015; 10 (1): 75-8.

39. Goldberg F, De Silvio A, Dreyer C. Radiographic assessment of simulated external root resorption cavities in maxillary incisors. Endod Dent Traumatol. 1998; 14 (3): 133-6.

40. Patel S, Dawood A, Wilson R, Horner K, Mannocci F. The detection and management of root resorption lesions using intraoral radiography and cone beam computed tomography - an in vivo investigation. Int Endod J. 2009; 42 (9): 831-8.

41. Madani Z, Moudi E, Bijani A, Mahmoudi E. Diagnostic accuracy of cone-beam computed tomography and periapical radiography in internal root resorption. Iran Endod J. 2016; 11 (1): 51-6.

42. White SC, Pharoah MJ. The evolution and application of dental maxillofacial imaging modalities. Dent Clin North Am. 2008; 52 (4): 689-705, v.

43. Kamburoglu K, Barenboim SF, Kaffe I. Comparison of conventional film with different digital and digitally filtered images in the detection of simulated internal resorption cavities--an ex vivo study in human cadaver jaws. Oral Surg Oral Med Oral Pathol Oral Radiol Endod. 2008; 105 (6): 790-7.

44. Holmes JP, Gulabivala K, van der Stelt PF. Detection of simulated internal tooth resorption using conventional radiography and subtraction imaging. Dentomaxillofac Radiol. 2001; 30 (5): 249-54.

45. Mohan RP, Verma S, Singh U, Agarwal N. Internal resorption. BMJ Case Rep. 2013. 
46. Celikten B, Uzuntas CF, Kurt H. Multiple idiopathic external and internal resorption: Case report with cone-beam computed tomography findings. Imaging Sci Dent. 2014; 44 (4): 315-20.

47. Darcey J, Qualtrough A. Resorption: part 2. Diagnosis and management. Br Dent J. 2013; 214 (10): 493-509.

48. Kamburoglu K. Evaluating root resorption lesions with CBCT. Am J Orthod Dentofacial Orthop. 2011; 140 (4): 453; author reply -4.

49. Kalender A, Oztan MD, Basmaci F, Aksoy U, Orhan K. CBCT evaluation of multiple idiopathic internal resorptions in permanent molars: case report. BMC Oral Health. 2014; 14: 39.

50. Qu X, Li G, Zhang Z, Ma X. Detection accuracy of in vitro approximal caries by cone beam computed tomography images. Eur J Radiol. 2011; 79 (2): e24-7.

51. Kamburoglu K, Murat S, Kolsuz E, Kurt H, Yuksel S, Paksoy C. Comparative assessment of subjective image quality of cross-sectional cone-beam computed tomography scans. J Oral Sci. 2011; 53 (4): 501-8.

52. Koc C, Sonmez G, Yilmaz F, Karahan S, Kamburoglu K. Comparison of the accuracy of periapical radiography with $\mathrm{CBCT}$ taken at 3 different voxel sizes in detecting simulated endodontic complications: an ex vivo study. Dentomaxillofac Radiol. 2018; 47 (4): 20170399.

53. Neves FS, Vasconcelos TV, Vaz SL, Freitas DQ, Haiter-Neto F. Evaluation of reconstructed images with different voxel sizes of acquisition in the diagnosis of simulated external root resorption using cone beam computed tomography. Int Endod J. 2012; 45 (3): 234-9.

54. Tayman MA, Kamburoglu K, Kucuk O, Ates FSO, Gunhan M. Comparison of linear and volumetric measurements obtained from periodontal defects by using cone beam-CT and micro-CT: an in vitro study. Clin Oral Investig. 2018.

55. Kolsuz ME, Bagis N, Orhan K, Avsever $\mathrm{H}$, Demiralp KO. Comparison of the influence of FOV sizes and different voxel resolutions for the assessment of periodontal defects. Dentomaxillofac Radiol. 2015; 44 (7): 20150070.

56. Librizzi ZT, Tadinada AS, Valiyaparambil JV, Lurie AG, Mallya SM. Cone-beam computed tomography to detect erosions of the temporomandibular joint: Effect of field of view and voxel size on diagnostic efficacy and effective dose. Am J Orthod Dentofacial Orthop. 2011; 140 (1): e25-30.

57. Ulusoy OI, Yilmazoglu MZ, Gorgul G. Effect of several thermoplastic canal filling techniques on surface temperature rise on roots with simulated internal resorption cavities: an infrared thermographic analysis. Int Endod J. 2015; 48 (2): 171-6. 